\title{
Case Report \\ Pasteurella Endocarditis: A Case Report and Statistical Analysis of the Literature
}

\author{
Randall S. Porter ${ }^{1}{ }^{1}$ and Christine M. Hay ${ }^{2}$ \\ ${ }^{1}$ University of Rochester School of Medicine and Dentistry, 601 Elmwood Ave, Rochester, NY 14642, USA \\ ${ }^{2}$ Department of Medicine, Infectious Diseases Division, University of Rochester Medical Center, 601 Elmwood Ave, Rochester, \\ NY 14642, USA \\ Correspondence should be addressed to Randall S. Porter; randall_porter@urmc.rochester.edu
}

Received 1 April 2020; Revised 16 June 2020; Accepted 24 June 2020; Published 20 July 2020

Academic Editor: Gernot Walder

Copyright (C 2020 Randall S. Porter and Christine M. Hay. This is an open access article distributed under the Creative Commons Attribution License, which permits unrestricted use, distribution, and reproduction in any medium, provided the original work is properly cited.

\begin{abstract}
Pasteurella is a genus of commensal bacteria of the oral cavity of several domesticated animals and a common cause of cellulitis after animal bites. Pasteurella has also been reported as a rare cause of endocarditis, with only 35 prior cases of definite Pasteurella endocarditis in the literature. Here, we present a case of Pasteurella multocida endocarditis treated successfully with surgery and antibiosis, as well as a review of the literature with statistical analysis of correlations between risk factors and clinical outcomes, as well as between treatment choices and clinical outcomes. Despite the small sample size, our analysis indicates a statistically significant correlation between comorbid liver disease and mortality, as well as a significant negative correlation between surgical treatment and mortality. This analysis implies a need for surgical management of endocarditis due to Pasteurella species and for more aggressive management of Pasteurella endocarditis in the setting of comorbid liver disease.
\end{abstract}

\section{Introduction}

Pasteurella is a genus of Gram-negative, facultative anaerobic, nonmotile bacilli which often exhibit pleomorphism and bipolar staining on microscopy. As indigenous flora of the oral cavity of many domesticated and tamed animals, including common pets and farm animals, they can be a relatively common cause of cellulitis after bites and scratches obtained from handling such animals. Endocarditis is a rare manifestation of Pasteurella infection and is typically due to Pasteurella multocida, often following known animal exposure. Only 35 cases of definite Pasteurella endocarditis have been reported prior to our case in the literature, primarily due to Pasteurella multocida. Due to its rarity and relatively high mortality, ongoing reporting of novel cases and analysis of the literature is vital to determining appropriate management of Pasteurella endocarditis.

\section{Case}

Our patient is a 66-year-old Caucasian woman with a complicated medical history of third-degree atrioventricular block ( $3^{\circ} \mathrm{AV}$ block) status post implantation of a Boston Scientific dual chamber internal cardioverter-defibrillator (ICD) with subpectoral pocket placement in Sep 2017, upgraded to dual-chamber ICD with right ventricular (RV) pacing lead extraction in Jan 2018, nonobstructive coronary artery disease (CAD) (50\% mid-left anterior descending artery (LAD) stenosis; $20 \%$ mid-right coronary artery (RCA) stenosis), ventricular tachycardia, severe aortic valve (AV) stenosis status post minimally-invasive \#21 On-X mechanical aortic valve replacement (AVR) in Sep 2017, thoracic aortic mycotic pseudoaneurysm status post repair in Oct 2017, hypertension, heart failure with preserved ejection fraction (HFpEF), chronic obstructive pulmonary disease (COPD) requiring $3 \mathrm{~L} / \mathrm{min} \mathrm{O}_{2}$, mild chronic normocytic anemia, chronic anxiety, and chronic depression. On Jul 23, 2019, the patient presented to our hospital with a 2-day history of decreased oral $(\mathrm{PO})$ intake, myalgias, nausea, bilious vomiting, night sweats, chills, diarrhea, lightheadedness, stool incontinence, and headache, as well as a 1-day history of chest pressure. Initial examination was positive for ventricular tachycardia, ill appearance, loud S1, and jugular venous 
distention (JVD). Pertinent findings in social history included a pet cat that sleeps in her bed at night but no known scratches or bites, a former smoking history of 50 pack-years, 1-2 cans of beer per night, and no illicit substance use. Initial labs indicated hyponatremia of $129 \mathrm{mEq} / \mathrm{L}$, a white blood cell count (WBC) of 11.8 cells $/ \mathrm{mm}^{3}$, negative serum troponins, a serum lactate of $2.4 \mathrm{U} / \mathrm{L}$, and a subtherapeutic international normalized ratio (INR) of 1.7. Chest X-ray (CXR) and computed tomography angiography (CTA) of the chest were negative for pathologic findings. She was started on an enoxaparin to warfarin bridge with a goal INR of 2-3, given intravenous (IV) fluid boluses, and had blood cultures drawn. On Jul 24, she was started on IV piperacillin-tazobactam $3.375 \mathrm{~g}$ every 8 hours (q8h). On Jul 26, she had increased work of breathing requiring bilevel positive airway pressure (BiPAP) for a few hours, and blood culture results indicated Pasteurella multocida, Staphylococcus epidermidis, and Staphylococcus warneri bacteremia. A transthoracic echocardiogram (TTE) was positive only for left atrial (LA) and left ventricular (LV) hypertrophy, aortic stenosis without regurgitation, moderate mitral regurgitation, mildly dilated ascending aorta, and moderately elevated pulmonary artery pressure, but no vegetations. On Jul 28, her piperacillintazobactam was discontinued and she was started on PO moxifloxacin $400 \mathrm{mg}$ daily. She was discharged on Jul 30 with continued moxifloxacin, the last dose of which was planned for Aug 6 to complete a total of 14 days of antibiotic treatment. The patient improved as an outpatient until the end of her antibiotic course, and an outpatient visit on Aug 19 revealed only occasional chills and fatigue, which resolved later that month. By Sep 9, however, she had developed aortic valve insufficiency, and on Sep 28, she developed shaking chills and altered mental status (AMS). On Sep 30, she presented to her primary care provider (PCP), who sent her to our emergency department (ED) for suspected sepsis.

On presentation to the ED, the patient displayed AMS, dyspnea with orthopnea, headache, and rigors, and was febrile to $38.5^{\circ} \mathrm{C}$, hypotensive at $80 / 57 \mathrm{mmHg}$, tachycardic at $114 \mathrm{BPM}$, and hypoxic to $75 \%$ oxygen saturation $\left(\mathrm{SpO}_{2}\right)$ on room air. Her CXR showed bilateral pulmonary edema, and labs were notable for a brain natriuretic peptide (BNP) of $12,000 \mathrm{pg} / \mathrm{mL}$, lactate of $3.8 \mathrm{U} / \mathrm{L}$, and serum $\mathrm{Na}$ of $123 \mathrm{mEq} / \mathrm{L}$. In the ED, she was placed on IV piperacillintazobactam $4.5 \mathrm{~g}$, decreased to $3.375 \mathrm{~g} \mathrm{q} 6 \mathrm{~h}$ after the first dose, IV azithromycin $500 \mathrm{mg}$ daily, IV doxycycline $100 \mathrm{mg}$ once, and IV linezolid $600 \mathrm{mg}$ once, as well as BiPAP with a positive end-expiratory pressure (PEEP) of $6 \mathrm{~cm} \mathrm{H}_{2} \mathrm{O}$ until her shortness of breath (SOB) improved. Azithromycin was discontinued on Oct 1 when blood cultures drawn on Sep 30 indicated Gram-negative bacteremia. Transesophageal echocardiogram (TEE) on Oct 2 indicated an aortic valve vegetation, as well as a paravalvular aneurysm on the aortic-mitral septum. By Oct 3, her bacteremia was identified as Pasteurella multocida. Repeat cultures were negative, and the patient clinically improved; she was discharged on Oct 8 with a continued course of IV piperacillin-tazobactam to be taken at home until Nov 5 . She continued to improve, and at an outpatient visit on Oct 21, she reported no chills, fever, chest pain, chest discomfort, or presyncope. At an outpatient visit on Oct 28, however, she was found to be severely dyspneic with an $\mathrm{SpO}_{2}$ of $81 \%$ on room air, with rales, wheezing, peripheral edema, and a diastolic murmur on exam. A TTE performed at that time indicated severe aortic regurgitation and a dilated right heart with severe tricuspid regurgitation, and she was readmitted.

On this admission, our patient described a gradual increase in SOB and home oxygen requirement, anorexia, and increased peripheral edema for 1 week, in addition to recent urinary hesitancy with oliguria. Exam indicated volume overload, and the patient was afebrile. Labs were notable for serum aspartate aminotransferase/alanine aminotransferase (AST/ALT) of 195/161 U/L, alkaline phosphatase of $208 \mathrm{U} / \mathrm{L}$, and a normal WBC count. She was continued on piperacillin-tazobactam, home torsemide, and $3 \mathrm{~L} / \mathrm{min} \mathrm{O}_{2}$. Her condition improved, and she remained stable until surgery on Nov 7 , which found prosthetic aortic valve endocarditis with an aortic root abscess extending into the RA and associated tricuspid annulus destruction. Bovine pericardium was used to repair the atrial perforation, cadaver allograft for a Bentall procedure, and a 33 Epic bioprosthetic valve for tricuspid replacement. Epicardial pacing wires were placed with the intent of replacement with dual-chamber ICD after resolution of her infection, and her chest was left open (due to difficulty mobilizing the chest wall) and packed and sealed with an iodine-impregnated incision drape $\left(\operatorname{Ioban}^{\mathrm{TM}}\right)$. Postoperatively, she required epinephrine, milrinone, vasopressin, and mechanical ventilation. Her WBC and temperature waxed and waned between elevated and normal for several days postoperatively, and hypoxia and hypercapnia required an increasing fraction of inspired oxygen $\left(\mathrm{FiO}_{2}\right)$. On Nov 9, she received a chest washout and primary chest closure. On Nov 10, initial culture from the tricuspid valve was positive for Staphylococcus warneri in addition to another organism later identified as a Curtobacterium sp.; in addition to her continued piperacillintazobactam, she was placed on IV daptomycin $8 \mathrm{mg} / \mathrm{kg}$ daily due to a questionable history of drug reaction with eosinophilia and systemic symptoms (DRESS) to vancomycin and contraindications (thrombocytopenia and anemia) to linezolid. She also was noted to have increasing abdominal distention at this time, and an abdominal CT showed dilated bowel with pneumobilia, which resolved over the next few days. On Nov 13, her S. warneri sensitivities indicated resistance to gentamicin and one colony to gentamicin and clindamycin but resistance in all colonies to cefazolin, erythromycin, linezolid, methicillin, oxacillin, penicillin $G$, tetracycline, and vancomycin; her daptomycin was discontinued at this time. She had return of bowel function on Nov 15. A possible infection of the right knee joint at this time resulted in the reinitiation of daptomycin for empiric methicillin-resistant Staphylococcus aureus (MRSA) coverage, discontinued on Nov 18 for lack of signs or symptoms concerning for joint infection. She was able to be weaned off vasopressor and inotrope support on Nov 16, and because of her development of ARDS and an expectation of prolonged intubation, she was 
given a tracheostomy and endotracheal tube on Nov 19 . Her WBC had been slowly declining over this period, and by Nov 20, she was no longer leukocytotic.

On Nov 21, she had return of abdominal pain and worsening distension, both of which had previously been resolving, and an abdominal XR indicated evolving ileus. Abdominal CT on Nov 22 found pneumatosis intestinalis and significant free air concerning for bowel perforation, and she was given a single dose of metronidazole before emergent exploratory laparotomy and small bowel resection for distal ileal obstruction. Postoperatively, she required norepinephrine for 1 day. She developed worsening abdominal pain and distension on Nov 24, and blood cultures drawn on Nov 21 were found to be positive for Gramnegative rods later identified as Klebsiella aerogenes; ciprofloxacin $400 \mathrm{mg}$ IV daily was started at this time, due to the patient's history of allergies to ceftriaxone and ertapenem. By Nov 27, return of bowel function was noted, repeat blood cultures had failed to grow any bacteria, and Klebsiella sensitivities indicated resistance to ampicillin, ampicillinsulbactam, cefazolin, ceftriaxone, and piperacillin-tazobactam, dose-dependent susceptibility to cefepime, and sensitivity to trimethoprim-sulfamethoxizole, tobramycin, meropenem, amikacin, and most importantly, ciprofloxacin, her last dose of which was planned for Nov 30 . However, her WBC elevated from 6.4 to 12.9 cells $/ \mathrm{mm}^{3}$ overnight, and by Nov 28, it was at 14.7 cells $/ \mathrm{mm}^{3}$ in addition to the development of abdominal distention and an abdominal XR indicating a dilated small bowel concerning for ileus and possible ischemia. A CT that day indicated free air and was concerning for an anastomotic leak; emergent surgery confirmed bowel contents in the peritoneum due to dehiscence of the anastomosis, which was resected prior to an ileostomy. Her piperacillin-tazobactam and ciprofloxacin were discontinued, and she was started on meropenem $2000 \mathrm{mg}$ q $8 \mathrm{~h}$.

On Nov 30, she became febrile and leukocytotic to 21.1 cells $/ \mathrm{mm}^{3}$, and her antibiotic regimen was adjusted to meropenem $500 \mathrm{mg}$ q6h, daptomycin $8 \mathrm{mg} / \mathrm{kg}$ daily, and fluconazole $400 \mathrm{mg}$ daily. She was afebrile by the following day, but her WBC count lowered only slightly over the next few days, and her fever had returned by Dec 6 . A CT on Dec 6 indicated two abdominal rim-enhancing loculated fluid collections concerning for abscesses. Her daptomycin was discontinued, and she received an abscess drainage of $9 \mathrm{~mL}$ of purulent fluid, drain placement, and paracentesis of $40 \mathrm{~mL}$ clear yellow to red fluid on Dec 7. She became afebrile, and her WBC decreased to 14.9 cells $/ \mathrm{mm}^{3}$, which continued to decrease over Dec 8 into the normal range. On Dec 10, surgical pathology of the resected bowel anastomosis was found to be consistent with cytomegalovirus (CMV) enteritis, and she was started on ganciclovir $5 \mathrm{mg} / \mathrm{kg}$ q12h on Dec 11 after a positive CMV quantitative polymerase chain reaction (qPCR) of $9419 \mathrm{IU} / \mathrm{mL}$. By Dec 11, her abscess fluid had also cultured Enterococcus faecium. Her meropenem was discontinued, and she was started on linezolid $600 \mathrm{mg}$ $\mathrm{q} 12 \mathrm{~h}$ and piperacillin-tazobactam $4.5 \mathrm{~g}$ q8h. Her WBC count continued to decrease and by Dec 15, she was leukocytopenic to 3.8 cells $/ \mathrm{mm}^{3}$. By Dec 17, her leukocytopenia had worsened to 2.8 cells $/ \mathrm{mm}^{3}$, and her ganciclovir was discontinued in favor of foscarnet $3500 \mathrm{mg}$ q12h. On Dec 19, her piperacillin-tazobactam was discontinued to complete her endocarditis treatment. An abdominal CT on Dec 23 showed complete resolution of her prior abscesses, and her linezolid was discontinued. Her abscess drains were removed on Dec 24, and her foscarnet was discontinued, as she had also completed her 14-day course of treatment for CMV enteritis.

Despite her recurrent infections, over the course of December our patient continued to slowly improve in respiratory and hemodynamic status. By Dec 22, she was ambulatory and could tolerate unassisted ventilation with tracheostomy trials of 3.5 hours, which improved rapidly over the next week to complete freedom from mechanical ventilation on Dec 28. On Dec 30, after over 48 hours off mechanical ventilation, she was given a Metronic automatic ICD, and her epicardial pacing wires were removed. By Jan 10,2020 , she was able to tolerate endotracheal tube capping of over 24 hours with good oxygenation, and her endotracheal tube was removed.

Our patient was discharged on Jan 13, 2020 to a skilled nursing facility (SNF) for rehabilitation. At the time of discharge, she had a well-functioning ileostomy requiring PO $\mathrm{NaCl}$ supplementation due to ileostomy losses, but otherwise enjoyed a regular diet. She was oxygenating well on room air after removal of her endotracheal tube, and her heart was well-paced by her ICD. After less than one week in her SNF, she was released home. In follow-up, she was seen by cardiothoracic surgery (Jan 23) and cardiology (Jan 29 and Feb 4). Over this time, paroxysmal atrial flutter was noticed in addition to one 15-second episode of ventricular tachycardia. On Feb 9, she was admitted to an out-of-network hospital for pneumonia which resolved after a short stay in the ICU; she was discharged home on Feb 18. She was seen again by cardiology on Mar 2 and cardiothoracic surgery on Mar 4 and showed improvement from her Feb 4 and Jan 29 cardiology appointments, with resolution of her atrial flutter. She had no further episodes of ventricular tachycardia, and her low appetite, low weight, fatigue, anxiety, and depression, which had worsened over the course of her most recent hospital stay, were all improving. As of Mar 30, 2020, she was continuing to improve and had no new health concerns.

\section{Literature Review}

3.1. Methods. We queried the PubMed database for all articles resulting from the search "Pasteurella" AND "endocarditis." All results were manually reviewed for relevance to our topic. Cases without microbiologically proven Pasteurella infection were excluded, as were cases which did not meet Duke or modified Duke criteria for the diagnosis of endocarditis. Articles in other languages were translated into English before review. Articles which met these criteria were examined for reference to cases previously described in the literature, and any such referenced articles novel to our search were reviewed in the same manner. After this search was completed, all articles which did not contain a novel case 
or otherwise add information which could not be extracted from the primary case literature were excluded. Each remaining case was examined, and pertinent information was extracted for statistical analysis.

In extracting information into a format useful for statistical analyses, some generalizations were made. Species of Pasteurella were not assumed to be that stated by each article at face value, particularly with respect to non-multocida species. Our earliest case presented to the hospital in 1962 [1], and many cases occurred prior to the molecular identification of Pasteurella species. Though distinction of Pasteurella from related genera, and typically distinction of P. multocida from non-multocida Pasteurella species, was feasible prior to the advent of these methods (papers which did not adequately categorize their infectious agents as definitively belonging to the Pasteurella genus having been excluded from our analysis), advancements have shown that even with current molecular methods, proper identification of non-multocida species remains challenging [2-10]. Therefore, we decided to categorize species as multocida and non-multocida, as many of the papers regarding P. dagmatis, $P$. pnemotropica, $P$. haemolytica, and $P$. ureae simply do not have enough information to retroactively verify the species identifications made. Likewise, to allow for easier and more robust statistical analysis of different factors' influence on case outcome, case outcome was reduced to a binary: death due to Pasteurella endocarditis or cure of endocarditis (regardless of sequelae); different categories of comorbidities were consolidated from individual diagnoses to broad categories (specifically, liver disease, heart disease, and substance abuse, as these were the most common broad categories of comorbidities across the literature).

Statistical analysis was carried out using Minitab ${ }^{\circledR} 19$. Each test was applied over all cases (including our own) which had adequate data regarding the hypothesis being questioned. We set the $\alpha$-value of each test to 0.05 prior to any analysis. Of the data extracted, we compared patient sex with the case outcome, as well as the species of Pasteurella (multocida versus nonmultocida) with the outcome, using Fisher's exact test, as the contingencies of the $\chi^{2}$ test of association were not met, and Fisher's exact test is preferable in cases of low expected cellvalues $[11,12]$. For comparison of the case outcome across comorbidities, $\chi^{2}$ contingencies were not met, and as we used three comorbidity categories (as previously discussed), a Fisher's exact test could not be applied to the data; therefore, while not ideal, we used the Fisher's exact test to test the association of each of the three comorbidity classes with case outcome individually. The association between surgical versus nonsurgical management and case outcome was evaluated using the Cochran-Mantel-Haenszel method to control for the potentially confounding variable of which valve was affected on the case outcome $[13,14]$. Finally, to counteract the possibility of finding falsely significant findings due to data mining, our raw $p$ values were corrected using the Benjamini-Hochberg method [15-17] at a (conservative) $q$ value of 0.05 .

3.2. Results. Including our current case, a total of 36 cases of adequately-verified Pasteurella endocarditis have been reported in the literature, a summary of which may be found in Table 1. Of these 36 cases, 24 were cases of Pasteurella multocida $[18,19,21,24,25,27,30-35,37-39,41-43$, $45,47-49$ ] and 12 were non-multocida species (reported as follows: $P$. dagmatis (4) [5, 20, 28, 36], P. pneumotropica (3) $[22,40,44], P$. haemolytica (3) $[1,26,46]$, and P. ureae (2) $[23,29]$; though as noted above, distinguishing between these species can be quite difficult, and thus, we do not take these species designations to be inherently accurate beyond their designation as non-multocida Pasteurella spp.). Males had a higher incidence, accounting for almost $2 / 3$ of the cases. The mean and median age at presentation were 56 and 57 , respectively, with a standard deviation of 17 years and a reasonably even distribution between ages 35 and 85 . Only one case occurred at an age $>85$ [42] and only 3 cases at an age $<35[22,25,44]$. Twenty-five cases (69\%) involved recent exposure to an animal, most commonly cats (17) $[5,18-21,28,30-32,39,41-43,45,49]$ and dogs (8) $[19,24,27,31,34,35,39,45]$, but also sheep (1) [38], rodents (1) [44], and fish (1) [44]. Of these, only 7 had a known history of animal bite [18, 20, 21, 28, 41, 42, 49], 2 with a known history of animal scratch [28, 40], 2 with a known history of an animal licking a wound $[5,27]$, and 3 with other wounds or indications of a local infectious nidus in the setting of animal exposure [18, 19, 35]; the other 12 cases of animal exposure occurred without any indication of a skin or soft tissue source [2, 24, 30-32, 34, 38, 39, 43-45]. The number of reported cases has increased over every decade since the first recorded case. Of those countries represented more than once, Saudi Arabia has reported 2 cases [26, 38], Japan $4[29,34,47,48]$, France $8[18,21,22,24,35,37,46]$, and the United States $13[2,5,19,20,23,27,28,31$, $32,36,43,44]$. While many patients presented with a past medical history $(\mathrm{PMH})$ significant for substance abuse (7, including ethanol and nicotine abuse) $[21,23,28,30,37$, $39,49]$, liver disease $(8)[18,21,25,28,30,31,35]$, or heart disease (17) $[2,5,18,20,25,27-29,31,32,34,36$, $40,41,44,45]$, other comorbidities were noted, though in 6 cases $[19,24,26,38,46,47]$, there was no significant $\mathrm{PMH}$ presented.

Systemic coinfections were reported only twice, once with Burkholderia cepaciae and Candida spp. in a cirrhotic French cat owner [18] and once with Streptococcus salivarius in a northwestern African with a history significant only for poor dentition and without known animal exposure [33]. While our patient presented initially with Staphylococcus epidermidis and Staphylococcus warneri coinfections, this was long before the patient began to show any signs, symptoms, or radiographic evidence of endocarditis, and these coinfections never resurfaced after initial antibiotic therapy. Resistance to antibiotics in Pasteurella strains was scarcely described across the literature. One study each reported resistance to penicillin [33] and piperacillintazobactam [48] in P. multocida and to bacitracin and triacetyloleandomycin in a non-multocida species [2]. Of the remaining studies, only three explicitly stated that no resistances were identified [23, 26, 29], and all other studies failed to discuss resistance, making an accurate antibiogram at this time impossible. All valves have been 


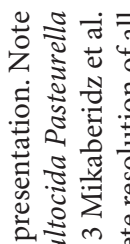

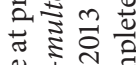

$\infty$ iิ

表

可

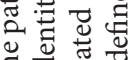

$\pm$

焉言

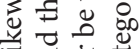

응 흥

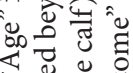

《

言

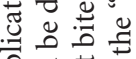

글 范

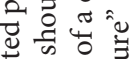

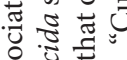

串

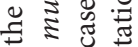

矛范

वे ते

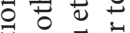

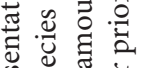

क्षे क्ष

氙 。

氙芯

पे 2 की

可㟔

है

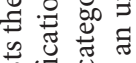

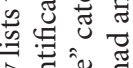

客票志

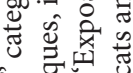

है

牙。

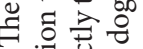

巡氙苛

政

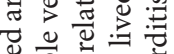

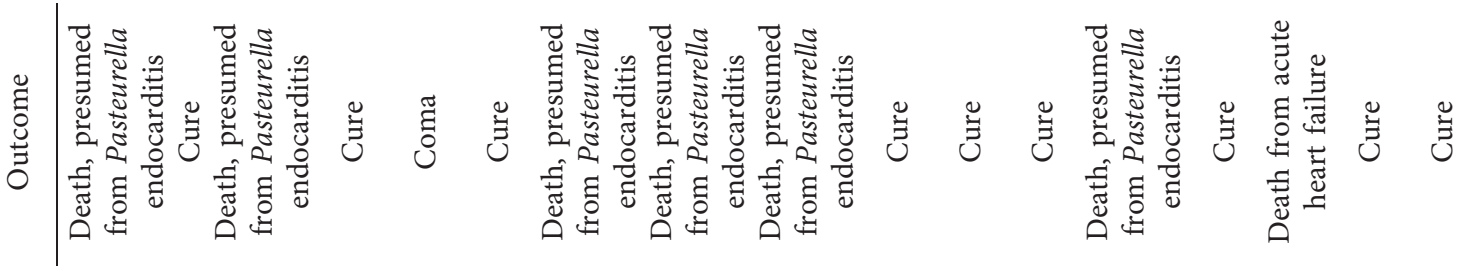

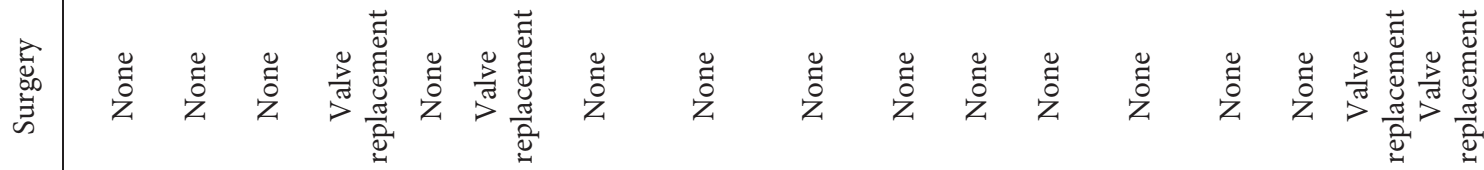

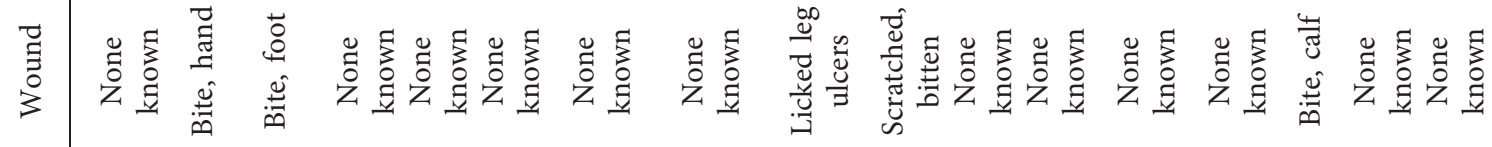

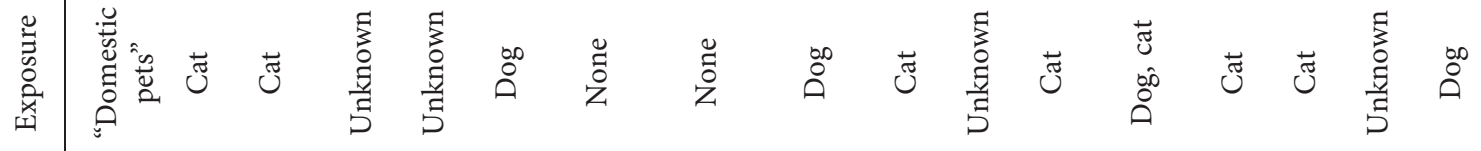

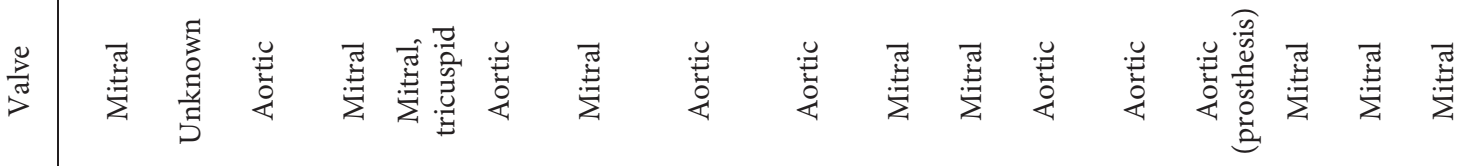

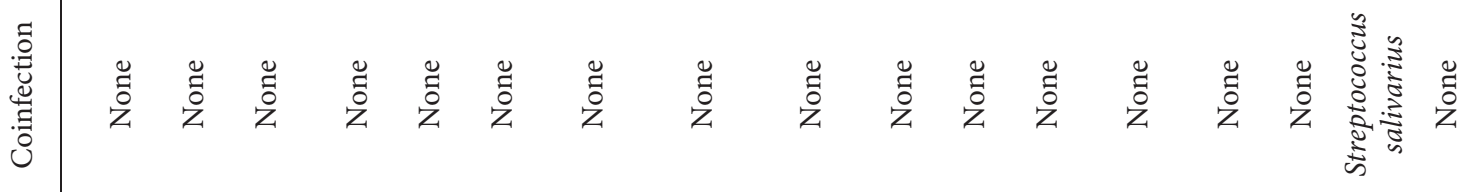

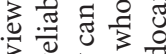

을

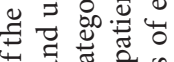

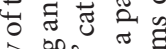

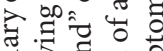

节言焉

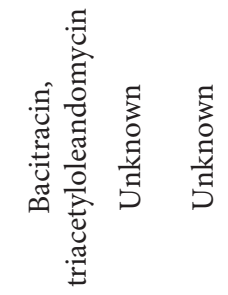

की 0 क्र क क

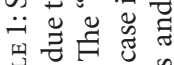

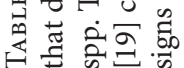

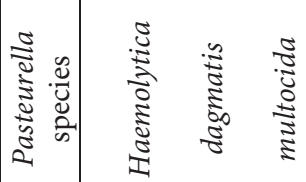

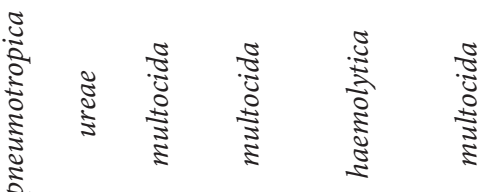

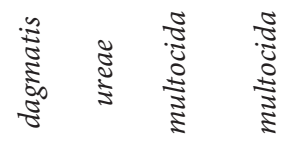

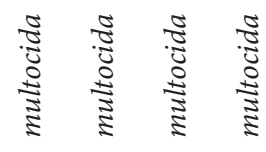

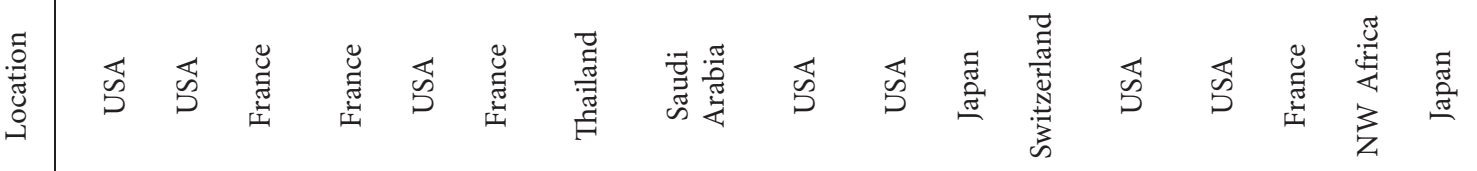

ॐึ山

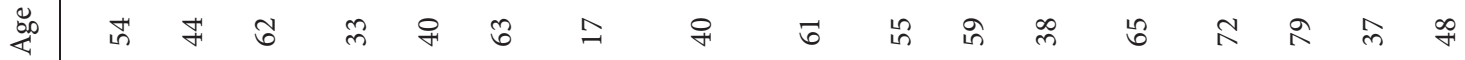
है

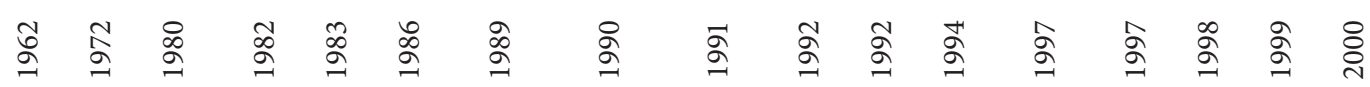

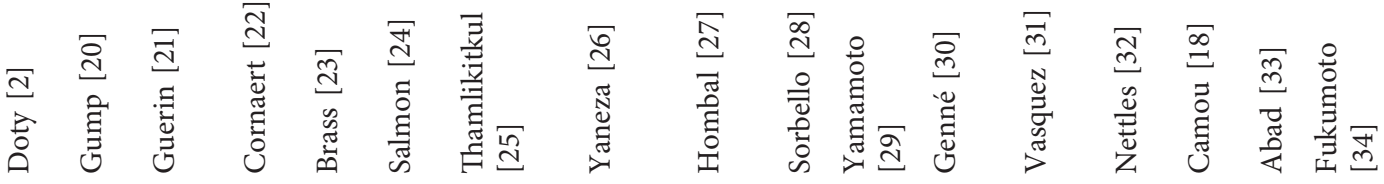




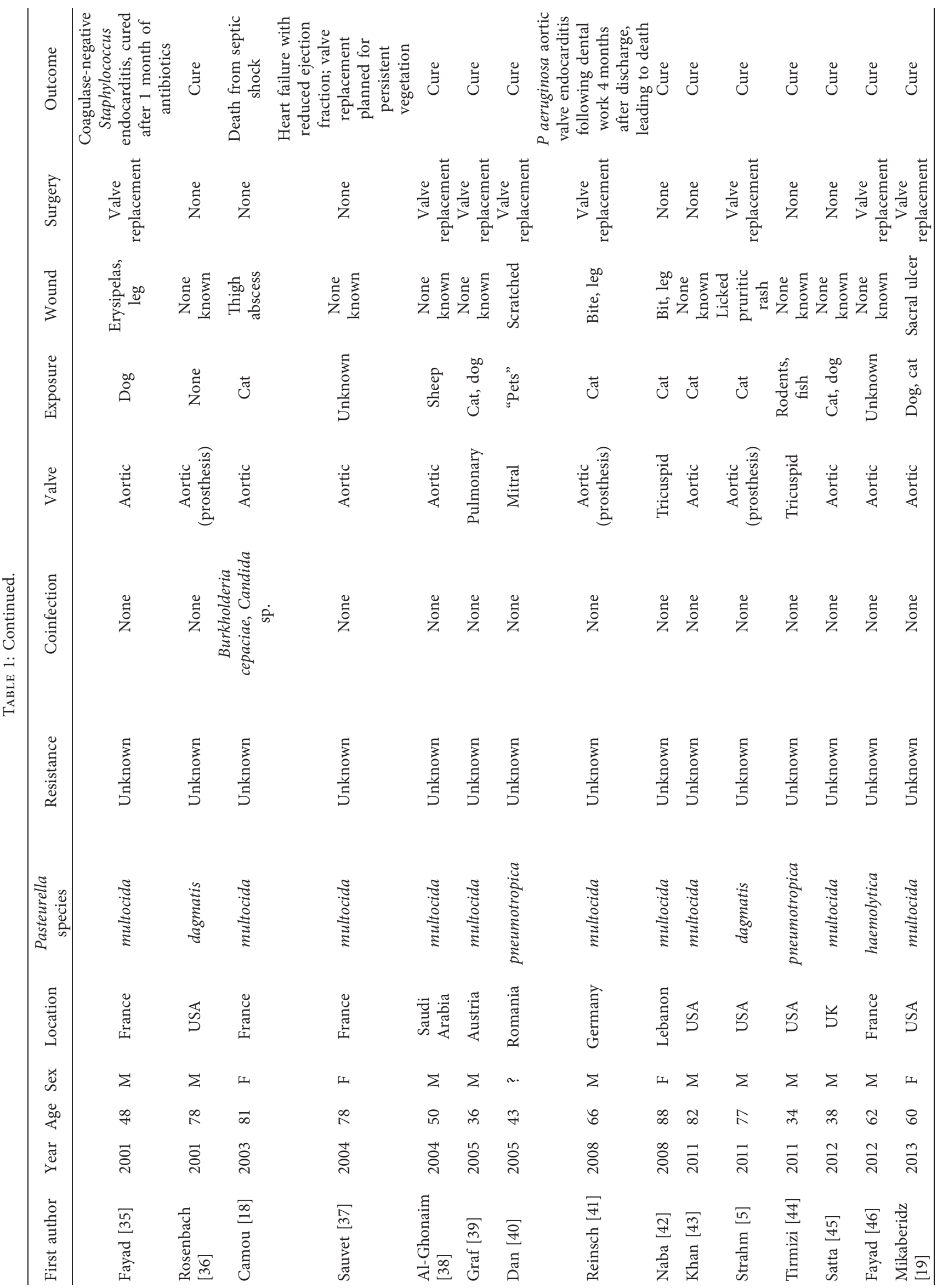




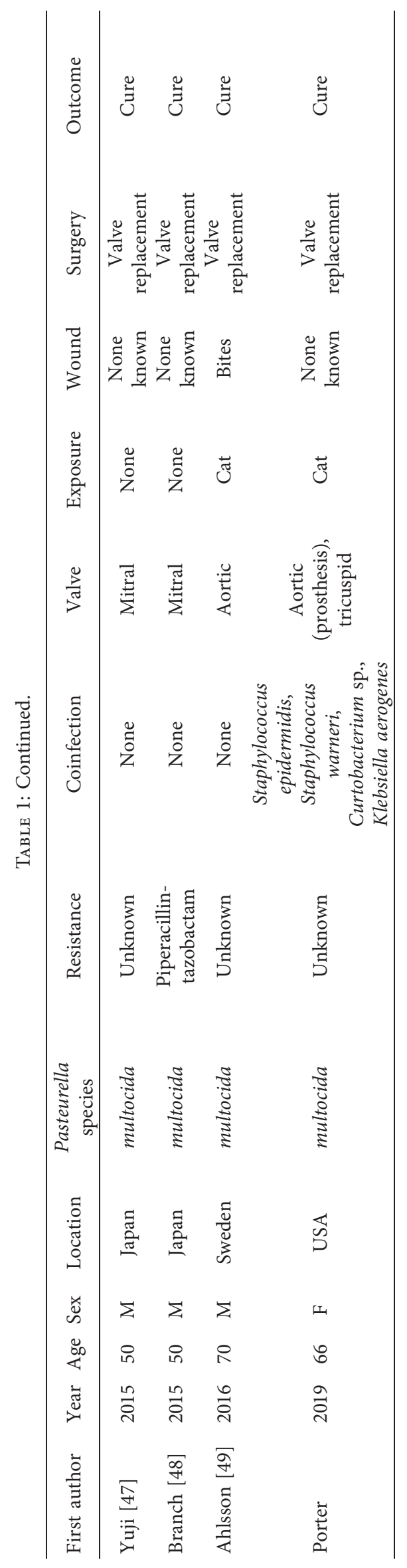


affected in the literature: 15 cases of native aortic valves $[18,19,21,24,26,27,30,31,35,37,38,43,45,46,49], 4$ prosthetic aortic valves (3 bioprostheses $[5,32,36]$ and 1 mechanical prosthetic [41]), 10 native mitral valves $[2,18,22,25,28,29,33,34,47,48], 2$ native tricuspid valves [42, 44], 1 native pulmonary valve [39], 1 dual-infection of native mitral and tricuspid valves [23], and our dual-infection of metal aortic prosthesis and native tricuspid valve. Complications and sequelae arose in most cases, which appeared primarily to be complications of sepsis and endocarditis or attributable to preexisting conditions, in many cases causing organ failure; only the reproductive system appeared spared from complications and sequelae across cases, though that may well be due to difficulty in acutely diagnosing unsuspected reproductive organ failure.

For treatment, all patients received antibiotics except for a patient who died shortly after presentation [21], typically with penicillins once culture and sensitivity results were available. Duration of antibiotic therapy varied widely both in cured patients and in those who died of their disease, often due to complications requiring elongation of the initiallyintended duration of therapy, but also due to differences in the initial choice of antibiotic therapy. We considered analyzing the association between duration of antibiotic therapy and case outcome, but due to the varied reasons for elongation or shortening of the antibiotic therapy duration across cases, it was decided that the results would have little utility due to the difficulties inherent in the interpretation of any potential results. In 16 cases, valve replacement was utilized for source control of the infection $[5,19,22,24,33-35,38-41,46-49]$. In total, 7 cases resulted in the patient's death during the course of endocarditis treatment, all of which occurred in the absence of surgical source control, representing $19 \%$ of all cases and 35\% of cases in which there was no surgical management of the affected valve $[2,18,21,25-27,31]$.

3.3. Statistical Analysis. Fisher's exact test between patient sex and case outcome was conducted due to a noted difference in the death rate between the sexes; this difference was not statistically significant ( $p$ values: 0.4327 raw/0.8654 corrected). Species ( $P$. multocida versus all other Pasteurella species studied as one group) was likewise not found to be significantly associated with case outcome ( $p$ values: 0.6910 raw/0.8292 corrected) using the same method. Individual Fisher's exact tests between the most prevalent comorbidities (liver disease, heart disease, and substance abuse) and case outcome resulted in $p$ values of 0.0064 raw/0.0385 corrected, $0.4338 \mathrm{raw} / 0.6508$ corrected, and $1.0000 \mathrm{raw} /$ 1.0000 corrected, respectively. This indicates that the observed increase in mortality in those with liver disease $(62.5 \%$ vs. $10.7 \%$ in those without liver disease) was statistically significant, while heart disease and substance abuse were not significantly associated with case outcome at our level of statistical power. Note that the $p$ value of the association between substance abuse and outcome is exactly 1 because the observed and expected values of each cell differed by $<1$. A Cochran-Mantel-Haenszel test of the association between surgical treatment and case outcome resulted in an odds ratio (OR) of dying after valve replacement compared to that with medical therapy alone of exactly 0 with a statistically significant $p$ value of $0.0093 \mathrm{raw} /$ 0.0278 corrected. Recall that the odds ratio is exactly 0 because no patients who received a valve replacement in the literature died of their endocarditis. Corrected $p$ values after Benjamini-Hochberg adjustment did not indicate any likely false discoveries at a conservative $q$ value of 0.05 . We initially intended to also test the association between the number of days from the onset of signs or symptoms and the surgical valve replacement with case outcome using logistic regression; however, since no patients treated surgically died in the course of treatment, the test is mathematically impossible to complete, leaving us with no information as to any possible connection between case outcome and the number of days between the onset of symptoms and valve replacement.

\section{Discussion}

Our case is notable as the only case in the literature to involve the aortic and tricuspid valves, only one of 2 cases involving multiple valves [23], and only one of 2 cases involving a mechanical prosthesis [41]. Our patient's recurrent course and multiple hospitalizations are likely due to seeding of her aortic prosthesis early in her first bout of sepsis, which then grew and was managed too conservatively in her second hospitalization. It took 110 days from the onset of symptoms to surgical intervention for source control of her endocarditis. Despite this prolonged clinical course, an extensive cardiac history, and multiple comorbidities, our case provides yet another example of the trend noted in the literature that all reported patients with Pasteurella endocarditis treated surgically have survived, while the overall mortality rate in reported patients without surgical intervention is $35 \%$.

Considering that only 36 cases of definite Pasteurella endocarditis exist in the literature (including the case presented here), it was not expected that our statistical analyses would have enough power to detect statistically significant results; it is quite possible that as the number of cases in the literature grows, further analyses will detect more statistically significant associations which may be of benefit in understanding typical progression of the disease and the most appropriate management. Considering the overall reported mortality of $19 \%$, it is impressive that none of the 16 patients on record who have received valve replacement surgery have died; likewise, the difference in mortality for those with comorbid liver disease, $62.5 \%$ versus $10.7 \%$ without liver disease, is striking. That these associations were found to be statistically significant even in such a small sample likely indicates a very strong correlation. While it has been vaguely suggested in the past that surgical source control is highly desirable in Pasteurella endocarditis, our results confirm this with strong statistical validity and suggest that (at least until further research can be conducted on appropriate management) valve replacement should be offered to all patients presenting with Pasteurella 
endocarditis who lack absolute contraindications to surgical intervention. It is unfortunate that the $100 \%$ survival after surgical intervention makes it mathematically impossible to evaluate any potential association between the duration of time between presentation to the hospital and valve replacement with case outcome; with the little data we have at present, we cannot determine when valve replacement should ideally be offered in the course of treating Pasteurella endocarditis, but simply that it should be offered. Considering the apparent success of valve replacement for source control across the literature, it is surprising that it was not offered to more than $44 \%$ of patients, though this is perhaps understandable given the multiple comorbidities, and thus risk factors for surgery, with which many of these patients presented. Still, it is our hope that the significance of our results leads to surgery being offered more consistently to Pasteurella endocarditis patients and, further, that patients with liver disease are watched especially closely for any sign of deterioration during the course of hospitalization for Pasteurella endocarditis. Pasteurella is a rare cause of endocarditis, and thus its ideal management has not yet been fully determined. Considering its high mortality risk, we must make full use of any significant associations we can glean from analysis of the literature, few though these associations may be at present.

\section{Data Availability}

The data used in our statistical analysis are a compilation of case data from Pasteurella endocarditis cases available in the peer-reviewed literature as cited in this manuscript and consist of patient characteristics, their treatment courses and clinical outcomes, and available information on the infecting bacterial species' characteristics. They are available by request from the corresponding author.

\section{Conflicts of Interest}

The authors declare that they have no conflicts of interest.

\section{Acknowledgments}

All funding was provided by the University of Rochester Infectious Diseases Division of the Department of Medicine.

\section{References}

[1] G. L. Doty, G. N. Loomus, and P. L. Wolf, "Pasteurella endocarditis," New England Journal of Medicine, vol. 268, no. 15 , pp. 830-832, 1963.

[2] P. Boerlin, H. H. Siegrist, A. P. Burnens et al., "Molecular identification and epidemiological tracing of Pasteurella multocida meningitis in a baby," Journal of Clinical Microbiology, vol. 38, no. 3, pp. 1235-1237, 2000.

[3] T. Guillard, V. Duval, R. Jobart et al., "Dog bite wound infection by Pasteurella dagmatis misidentified as Pasteurella pneumotropica by automated system Vitek 2," Diagnostic Microbiology and Infectious Disease, vol. 65, no. 3, pp. 347-348, 2009.

[4] T. Akahane, M. Nagata, T. Matsumoto et al., "A case of wound dual infection with Pasteurella dagmatis and Pasteurella Canis resulting from a dog bite-limitations of Vitek-2 system in exact identification of Pasteurella species," European Journal of Medical Research, vol. 16, no. 12, pp. 531-536, 2011.

[5] C. Strahm, D. Goldenberger, M. Gutmann, P. Kuhnert, and P. Graber, "Prosthetic valve endocarditis caused by a Pasteurella dagmatis-like isolate originating from a patient's cat," Journal of Clinical Microbiology, vol. 50, no. 8, pp. 2818-2819, 2012.

[6] S. Zangenah, G. Güleryüz, S. Boräng, M. Ullberg, P. Bergman, and V. Özenci, "Identification of clinical Pasteurella isolates by MALDI-TOF-a comparison with VITEK 2 and conventional microbiological methods," Diagnostic Microbiology and Infectious Disease, vol. 77, no. 2, pp. 96-98, 2013.

[7] J. Xiong, S. Krajden, J. V. Kus et al., "Bacteremia due to Pasteurella dagmatis acquired from a dog bite, with a review of systemic infections and challenges in laboratory identification," Canadian Journal of Infectious Diseases and Medical Microbiology, vol. 26, no. 5, pp. 273-276, 2015.

[8] H. Shirzad-Aski and M. Tabatabaei, "Molecular characterization of Pasteurella multocida isolates obtained from poultry, ruminant, cats and dogs using RAPD and REP-PCR analysis," Molecular Biology Research Communications, vol. 5, no. 3, pp. 123-132, 2016.

[9] A. Pujiono, I. W. T. Wibawan, U. Afiff, and S. Setiyaningsih, "Molecular identification and serogrouping of Pasteurella mutocida field isolats," IOP Conference Series: Earth and Environmental Science, vol. 197, Article ID 012046, 2018.

[10] M. Orynbayev, K. Sultankulova, A. Sansyzbay et al., "Biological characterization of Pasteurella multocida present in the Saiga population," BMC Microbiology, vol. 19, no. 1, p. 37, 2019.

[11] R. A. Fisher, "On the interpretation of $\chi^{2}$ from contingency tables, and the calculation of P," Journal of the Royal Statistical Society, vol. 85, no. 1, pp. 87-94, 1922.

[12] A. Agresti, "A survey of exact inference for contingency tables,” Statistical Science, vol. 7, no. 1, pp. 131-153, 1992.

[13] W. G. Cochran, "Some methods for strengthening the common $\chi^{2}$ tests," Biometrics, vol. 10, no. 4, pp. 417-451, 1954.

[14] N. Mantel and W. Haenszel, "Statistical aspects of the analysis of data from retrospective studies of disease," Journal of the National Cancer Institute, vol. 22, no. 4, pp. 719-748, 1959.

[15] Y. Benjamini and Y. Hochberg, "Controlling the false discovery rate: a practical and powerful approach to multiple testing," Journal of the Royal Statistical Society: Series B (Methodological), vol. 57, no. 1, pp. 289-300, 1995.

[16] Y. Benjamini, A. M. Krieger, and D. Yekutieli, "Adaptive linear step-up procedures that control the false discovery rate," Biometrika, vol. 93, no. 3, pp. 491-507, 2006.

[17] Y. Benjamini, "Discovering the false discovery rate," Journal of the Royal Statistical Society: Series B (Statistical Methodology), vol. 72, no. 4, pp. 405-416, 2010.

[18] F. Camou, O. Guisset, S. Pereyre et al., "Endocardites à Pasteurella sp. Deux cas," Médecine et Maladies Infectieuses, vol. 35, no. 11, pp. 556-559, 2005.

[19] N. Mikaberidz, E. Y. Li, and C. C. Taub, "Pasteurella multocida infective endocarditis in an immunocompetent patient complicated by rhabdomyolysis and permanent hearing loss," Journal of Cardiovascular Disease Research, vol. 4, no. 1, pp. 55-57, 2013.

[20] D. W. Gump and R. A. Holden, "Endocarditis caused by a new species of Pasteurella," Annals of Internal Medicine, vol. 76, no. 2, pp. 275-278, 1972. 
[21] J. M. Guerin, J. M. Segrestaa, and M. Lamotte, "Pasteurella multocida endocarditis," La Nouvelle Presse Medicale, vol. 9, no. 37, pp. 2735-2736, 1980.

[22] P. Cornaert, P. Masson, G. Forzy et al., "Infectious endocarditis caused by rare germs. Review of the literature apropos of 2 cases," Annals of Cardiology and Angiology, vol. 36, no. 2, pp. 87-93, 1987.

[23] E. P. Brass, L. M. Wray, and T. McDuff, "Pasteurella ureae meningitis associated with endocarditis. Report of a case and review of the literature," European Neurology, vol. 22, no. 2, pp. 138-141, 1983.

[24] D. Salmon, B. Fantin, F. Bricaire, J.-L. Vilde, B. Pangon, and D. Ferand, "Endocarditis due to Pasteurella multocida with glomerulonephritis," The American Journal of Medicine, vol. 86, no. 4, p. 493, 1989.

[25] V. Thamlikitkul and T. Sangruchi, "Pasteurella multocida infective endocarditis: a case report," Journal of the Medical Association of Thailand = Chotmaihet Thangphaet, vol. 73, no. 12, pp. 704-706, 1990.

[26] A. L. Yaneza, H. Jivan, P. Kumari, and M. S. Togoo, "Pasteurella haemolytica endocarditis," Journal of Infection, vol. 23, no. 1, pp. 65-67, 1991.

[27] S. M. Hombal and H. P. Dincsoy, "Pasteurella multocida endocarditis," American Journal of Clinical Pathology, vol. 98, no. 6, pp. 565-568, 1992.

[28] A. F. Sorbello, J. O’Donnell, J. Kaiser-Smith, J. Fitzharris, J. Shinkarow, and S. Doneson, "Infective endocarditis due to Pasteurella dagmatis: case report and review," Clinical Infectious Diseases, vol. 18, no. 3, pp. 336-338, 1994.

[29] K. Yamamoto, U. Ikeda, C. Ogawa, H. Fukazawa, M. Eto, and K. Shimada, "Pasteurella ureae endocarditis," Internal Medicine, vol. 32, no. 11, pp. 872-874, 1993.

[30] D. Genne, H. H. Siegrist, P. Monnier, M. Nobel, L. Humair, and A. d. Torrente, "Pasteurella multocida endocarditis: report of a case and review of the literature," Scandinavian Journal of Infectious Diseases, vol. 28, no. 1, pp. 95-97, 1996.

[31] J. E. Vasquez, D. A. Fergusonr Jr., S. Bin-Sagheer et al., "Pasteurella multocida endocarditis: a molecular epidemiological study," Clinical Infectious Diseases, vol. 26, no. 2, pp. 518-519, 1998.

[32] R. E. Nettles and D. J. Sexton, "Pasteurella multocida Prosthetic valve endocarditis: case report and review," Clinical Infectious Diseases, vol. 25, no. 4, pp. 920-921, 1997.

[33] C. Abad, J. J. Cáceres, J. M. Ferrer, L. González, F. Álvarez, and A. Bordes, "Pasteurella multocida empyema in a patient after mitral valve replacement secondary to endocarditis," Anales de Medicina Interna, vol. 20, no. 8, pp. 441-442, 2003.

[34] Y. Fukumoto, Y. Moriyama, Y. Iguro, R. Toda, and A. Taira, "Pasteurella multocida endocarditis: report of a case," Surgery Today, vol. 32, no. 6, pp. 513-515, 2002.

[35] G. Fayad, T. Modine, S. Mokhtari et al., "Pasteurella multocida aortic valve endocarditis: case report and literature review," The Journal of Heart Valve Disease, vol. 12, no. 2, pp. 261-263, 2003.

[36] K. A. Rosenbach, J. Poblete, and J. Larkin, "Prosthetic valve endocarditis caused by Pasteurella dagmatis," Southern Medical Journal, vol. 94, no. 10, pp. 1033-1035, 2001.

[37] F. Sauvet, B. Graffin, S. Cremades et al., "Endocardite à Pasteurella multocida révélée par des rachialgies inflammatoires," La Revue de Médecine Interne, vol. 25, no. 7, pp. 530-531, 2004.

[38] M. A. Al Ghonaim, A. A. Abba, and M. Al Nozha, "Endocarditis caused by Pasteurella multocida," Annals of Saudi Medicine, vol. 26, no. 2, pp. 147-149, 2006.
[39] S. Graf, T. Binder, M. Heger, P. Apfalter, N. Simon, and S. Winkler, "Isolated endocarditis of the pulmonary valve caused by Pasteurella multocida," Infection, vol. 35, no. 1, pp. 43-45, 2007.

[40] M. Dan, C. Prisacariu, G. I. Georgescu, C. GeorgescuArsenescu, G. Tinica, and D. Buiuc, "Subacute bacterial endocarditis due to Pasteurella pneumotropica. Case Report," Revista medico-chirurgicala Societatii de Medici si Naturalisti din Iasi, vol. 109, no. 4, pp. 743-745, 2005.

[41] N. Reinsch, B. Plicht, A. Lind et al., "Recurrent infective endocarditis with uncommon Gram-negative Pasteurella multocida and Pseudomonas aeruginosa: a case report," The Journal of Heart Valve Disease, vol. 17, no. 6, pp. 710-713, 2008.

[42] M. R. Naba, G. F. Araj, Z. A. Kanafani, and S. S. Kanj, "First case of Pasteurella multocida endocarditis of the tricuspid valve: a favorable outcome following medical treatment," International Journal of Infectious Diseases, vol. 13, no. 5, pp. e267-e269, 2009.

[43] M. F. Khan, M. R. Movahed, and J. Jung, "Pasteurella multocida endocarditis," The Journal of Heart Valve Disease, vol. 21, no. 2, pp. 260-262, 2012.

[44] A. Tirmizi, S. Butt, and S. Molitorisz, "First reported case of Pasteurella pneumotropica tricuspid valve endocarditis," International Journal of Cardiology, vol. 161, no. 3, pp. e44e45, 2012.

[45] G. Satta, R. L. Gorton, and H. Kandil, "Prosthetic valve endocarditis caused by Pasteurella in a penicillin allergic patient: challenges in diagnosis and treatment," Infectious Disease Reports, vol. 4, no. 2, p. e32, 2012.

[46] G. Fayad, T. Modine, M. Koussa, E. Senneville, and O. Leroy, "First documented surgical case of human aortic valve endocarditis caused by Pasteurella haemolytica," The Journal of Heart Valve Disease, vol. 21, no. 1, p. 139, 2012.

[47] D. Yuji, M. Tanaka, Katayama, and K. Noguchi, "Pasteurella multocida infective endocarditis," The Journal of Heart Valve Disease, vol. 24, no. 6, pp. 778-779, 2015.

[48] J. Branch, T. Kakutani, S. Kuroda, Y. Shiba, and I. Kitagawa, "Pasteurella multocida infective endocarditis: a possible link with primary upper respiratory tract infection," Internal Medicine, vol. 54, no. 24, pp. 3225-3231, 2015.

[49] A. Ahlsson, Ö. Friberg, and J. Källman, "An angry cat causing Pasteurella multocida endocarditis and aortic valve replacement-A case report," International Journal of Surgery Case Reports, vol. 24, pp. 91-93, 2016. 